Ann. Sci. forest., 1969, 26 (4), 511-517.

\title{
IDENTIFICATION DE L'ACIDE ABSCISIQUE DANS LES BOURGEONS DORMANTS DE PINUS SYLVESTRIS
}

\author{
M. BONNET-MASIMBERT (1)
}

Laboratoire de Physiologie pluricellulaire, Centre national de la Recherche scientifique, 91 -Gif-sur-Yvette

\section{SOMMAIRE}

La concentration d'un inhibiteur de croissance présent dans les bourgeons dormants de Pinus sylvestris diminue fortement au moment du débourrement. Les propriétés physiologiques et chromatographiques de cet inhibiteur sont semblables à celles de l'acide abscisique.

Après plusieurs chromatographies sur colonne et couches minces, l'inhibiteur s'est montré être, par chromatographie en phase gazeuse, identique à l'acide abscisique. Une estimation basée sur des tests biologiques, montre que la concentration d'acide abscisique est relativement élevée pendant la phase de dormance (environ $4 \mathrm{mg}$ par $\mathrm{kg}$ de matériel frais).

\section{INTRODUCTION}

En matière forestière, les phénomènes de dormance prennent une importance particulière puisqu'il s'agit de plantes perennes. La sortie de dormance au printemps, en particulier, représente une étape critique à cause des gelées tardives qui peuvent survenir.

Au cours d'une étude préliminaire, nous avons pu montrer qu'une forte diminution dans la concentration d'un inhibiteur de croissance accompagnait les premières manifestations externes du débourrement. Dans la présente étude, nous avons cherché à déterminer la nature de cet inhibiteur.

(1) Adresse actuelle: Station d'Amélioration des Arbres forestiers, I.N.R.A., 14, rue Girardet. 54 - Nancy. 


\section{MATERIEL ET TECHNIQUES}

Nous avons utilisé $300 \mathrm{~g}$ de bourgeons de Pinus sylvestris récoltés quinze jours environ avant la date du débourrement. Nous avons procédé à une extraction de ce matériel, suivie de plusieurs séparations chromatographiques. Au cours des étapes successives de purification, l'activité biologique a toujours été mesurée au moyen du test «coléopti'e de blé » de NiTSCH et Nitsch (1956).

\section{KESULTATS EXPERIMENTAUX}

\section{1. - Extraction}

Nous avons effectué l'extraction sur les bourgeons entiers, sans éliminer les écailles protectrices, au moyen de méthanol à $80 \%$, après broyage fin dans un mortier, en présence de sable de Fontainebleau lavé aux acides. Cette extraction a eu lieu en chambre froide $\left(3^{\circ} \mathrm{C}\right)$, pour limiter les phénomènes d'oxydation.

\section{2. - Purification de l'extrait}

a) Purification par solvants non miscibles.

Après évaporation du méthanol sous pression réduite, l'extrait a été repris par une solution aqueuse de bicarbonate de sodium $(100 \mathrm{~g} / \mathrm{l})$ et extrait dans une ampoule à décanter avec de l'acétate d'éthyle. Après trois épuisements de la phase aqueuse, la phase acétate a été éliminée. La phase aqueuse a été ramenée à $\mathrm{pH} 2,5 \mathrm{par} \mathrm{HCl}$, puis extraite 4 fois avec de l'acétate d'éthyle neuf. La phase organique a été évaporée sous pression réduite à $30^{\circ} \mathrm{C}$. Nous avons ainsi obtenu l'extrait acide A.

b) Adsorption sur charbon actif.

Une quantité d'extrait A correspondant à $150 \mathrm{~g}$ de bourgeons a été reprise par $3 \mathrm{ml}$ d'eau, puis par $0,3 \mathrm{ml}$ de méthanol pur. Selon la technique utilisée par Davis (1968), nous avons placé l'extrait sur une colonne $(20 \mathrm{~cm} \times 2,5 \mathrm{~cm})$ contenant $6 \mathrm{~g}$ de Célite mélangée à $3 \mathrm{~g}$ de charbon actif. L'élution a été effectuée d'abord par $50 \mathrm{ml}$ d'eau, puis par $200 \mathrm{ml}$ d'acétone à $60 \%$.

Après élimination des 50 premiers $\mathrm{ml}$, nous avons recueilli 8 fractions de $25 \mathrm{ml}$ chacune. Un échantillon $(0,5 \mathrm{ml})$ de chaque fraction a été séché sous vide puis testé au test coléoptile de blé. Dans la fraction $\mathrm{n}^{\circ} 1$, une inhibition totale accompagnée d'une très forte toxicité (coléoptiles devenus flasques) a été décelée. Dans la fraction $\mathrm{n}^{\circ} 2$, la toxicité était moins forte. Les fractions $\mathrm{n}^{\circ} 3,4$ et 5 présentaient une inhibition totale mais dépourvue d'effets toxiques. Dans les dernières fractions enfin, l'inhibition devenait faible, puis nulle.

Nous avons regroupé les fractions $n^{\circ} 3,4$ et 5 qui ont été séchées sous pression réduite et constituent l'extrait $\mathrm{B}$. Une chromatographie simultanée d'une fraction des extraits $\mathrm{A}$ et $\mathrm{B}$, sur papier Whatman $3 \mathrm{~mm}$ dans le solvant isopropanol-ammoniaqueeau $(80: 0,5: 19,5)$ et observée sous U.V. $(254 \mathrm{~m} \mu)$ a montré que l'extrait B était 
nettement purifié par rapport à l'extrait A mais contenait encore une bande fortement inhibitrice entre les $\mathrm{Rf} 0,55$ et 0,75 .

c) Purification en chromatographie sur couches minces.

Nous avons alors pratiqué plusieurs chromatographies successives sur plaques de Silicagel-Merck HF-254, contenant un produit fluorescent. Nous avons utilisé certains des solvants adoptés par RUDNICKI (1969).

Après chaque chromatographie, nous avons élué la bande absorbante violette aux U.V. (254 $\mathrm{m} \mu$ ) située exactement en face d'un témoin d'acide abscisique (ABA) chromatographié en même temps. L'éluat de cette zone a été rechromatographié dans un autre solvant selon la séquence :

extrait $\mathrm{B}$ dans : benzène-acétone-ac. acétique $(70: 30: 1)$;

$\rightarrow$ on recueille la bande $\mathrm{C}$ de $\mathrm{Rf} 0,46$,

bande $\mathrm{C}$ dans : benzène-acétate d'éthyle-ac. acétique $(70: 30: 5)$;

$\rightarrow$ on recueille la bande D de Rf 0,17 ,

bande $\mathrm{D}$ dans : benzène-chloroforme-ac. acétique $(50: 50: 0,5)$.

Pour cette dernière chromatographie, nous avons procédé à 7 montées successives, sur $15 \mathrm{~cm}$, du même chromatogramme de D. En effet, dans ce solvant, la migration de l'ABA est très faible, mais non nulle. Ceci permet d'éliminer dans l'extrait $D$ un certain nombre de substances migrant plus rapidement que l'ABA ainsi que celles qui restent au niveau du spot de départ. Après ces opérations, la tache d'ABA se trouve à $1 \mathrm{~cm}$ au-dessus du spot. La bande de l'extrait $\mathrm{D}$ située en face de cette tache constitue l'échantillon E.

Après chaque chromatographie, le spectre U.V. de l'éluat de la zone inhibitrice a été déterminé au spectrophotomètre Beckman, ainsi que celui du témoin d'ABA dans le même système. Nous avons ainsi pu suivre la simplification du spectre, en particulier, la disparition d'un pic voisin de $300 \mathrm{~m} \mu$ sur l'extrait très purifié E qui, cependant, continue à inhiber très fortement l'élongation des coléoptiles de blé. Au cours de ces opérations, l'ABA ne subit aucune modification de spectre.

\section{3. - Identification par chromatographie en phase gazeuse}

L'identification du composé actif de l'extrait E a été effectuée par le Dr. B. H. Most par chromatographie en phase gazeuse selon la technique qu'il a mise au point (Most, 1969) et qui est proche de celle utilisée par MacMillan et coll. (1968) pour les gibbérellines.

L'analyse a été effectuée sur un appareil F. et M. 402 (F and M Scientific Corp., Avondale, $\mathrm{Pa}$.) avec double colonne à injection directe et double détecteur à ionisation de flamme permettant des analyses isothermes avec l'hélium comme gaz vecteur.

Les analyses ont été effectuées sur deux types de colonnes :

- colonne A : $2 \%$ QF-1 sur Gas-chrom A (80-100 mesh) à $187^{\circ} \mathrm{C}$.

- colonne B : $2 \%$ SE-33 sur Chromosorb G (80-100 mesh) à $167^{\circ} \mathrm{C}$.

Avant chromatographie, l'échantillon E ainsi qu'un témoin d'ABA ont été méthylés avec du diazométhane dans du méthanol. Ils ont ensuite été séchés puis repris pour analyse par $30 \mu \mathrm{l}$ de méthanol 
La figure 1 présente les enregistrements obtenus sur les deux colonnes pour l'échantillon $\mathrm{E}$ ainsi que pour le témoin. Le tableau $\mathrm{I}$ indique la valeur des temps de rétention des pics susceptibles de correspondre à l'acide abscisique.

\section{Tableau I}

Comparaison des temps ide rétention en chromatographie en phase gazeuse de l'acide abscisique et de l'inhibiteur du Pin

\begin{tabular}{|c|c|c|}
\hline \multirow{2}{*}{$\begin{array}{l}\text { Echantillon testé } \\
\text { (après méthylation) }\end{array}$} & \multicolumn{2}{|c|}{ Temps de rétention (en minutes) } \\
\hline & $\begin{array}{c}\text { Colonne } 2 \% \mathrm{QF}-1 \\
187^{\circ} \mathrm{C}\end{array}$ & $\begin{array}{c}\text { Co'onne } 2 \% \text { SE-33 } \\
167^{\circ} \mathrm{C}\end{array}$ \\
\hline$\ldots \ldots \ldots \ldots \ldots \ldots \ldots$ & 3,85 & 10,66 \\
\hline Inhibiteur de Pinus ........... & 3,90 & 10,66 \\
\hline
\end{tabular}

On constate que, sur la colonne à $2 \%$ de SE-33, il y a une parfaite identité entre l'acide abscisique et l'un des pics de l'inhibiteur du pin. Sur la colonne à $2 \%$ de QF-1, l'analogie, bien que moins parfaite, semble confirmer le premier résultat.

\section{DISCUSSION}

Les travaux de MacMillan et coll. (1968) pour les gibérellines et de Most (1969) pour l'acide abscisique ont montré que la chromatographie en phase gazeuse constitue une méthode sensible et précise d'identification de ces régulateurs de croissance. Les résultats obtenus avec l'inhibiteur du pin, du point de vue physiologique (inhibition de l'élongation de segments de coléoptiles de blé), du point de vue de la chromatographie sur couches minces (même Rf que l'ABA dans 3 solvants) ou de celle en phase gazeuse (sur deux types de colonnes) montrent de façon claire qu'il s'agit, ici encore, de l'acide abscisique.

Le rôle de l'acide abscisique dans les phénomènes de dormance des arbres a été mis en lumière par les travaux de WAREING et coll. (1967) après que la «dormine », isolée par WAREING et coll. (1963) à partir de feuilles d'érable récoltées à la fin de l'été, ait été identifiée par CoRNFORTH et coll. (1965) à l'acide abscisique ou «abscisine II » isolée par AdDicotr et coll. (1963). La présence d'un inhibiteur dans les extraits de Pinus sylvestris avait déjà été signalée par Michniewicz (1967). Commie cet auteur, nous avons observé une baisse du taux de l'inhibiteur naturel au début du débourrement. Dans les bourgeons dormants, la concentration d'inhibiteur est relativement élevée. D'après l'activité biologique des extraits, nous avons pu calculer qu'elle devrait être d'environ $4 \mathrm{mg}$ d'ABA par $\mathrm{kg}$ de poids frais.

Le rôle exact des inhibiteurs naturels en tant que régulateurs de la croissance et de la dormance des arbres est encore mal élucidé. Dès 1959, NiTsCH et NiTsCH attiraient l'attention sur le fait que les changements photopériodiques qui entraînent l'entrée en dormance conduisent au remaniement des niveaux des substances stimulant la 

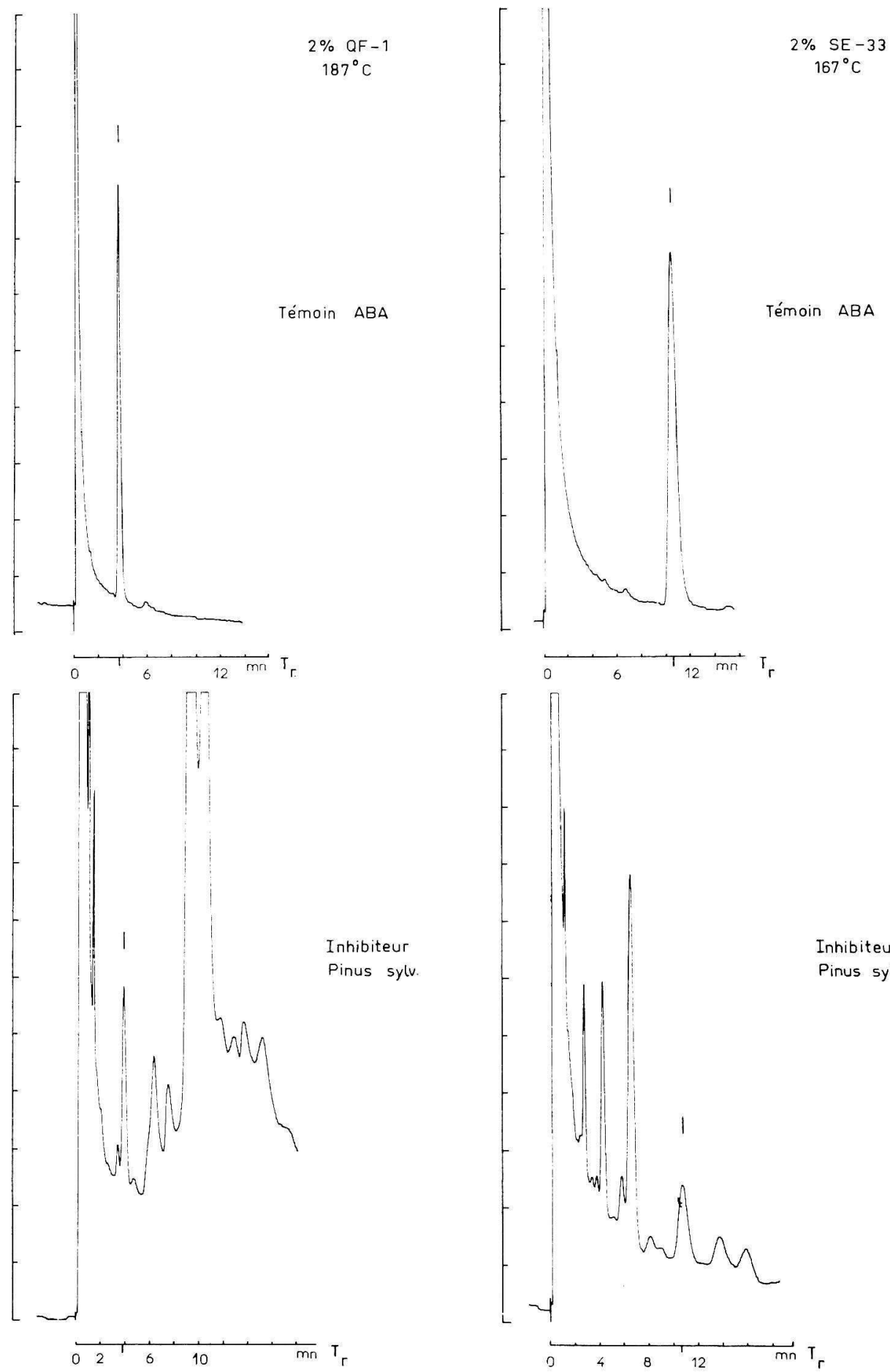

\% QF - 1

$187^{\circ} \mathrm{C}$

Témoin $A B A$

TBA

Inhibiteur

Pinus sylv.
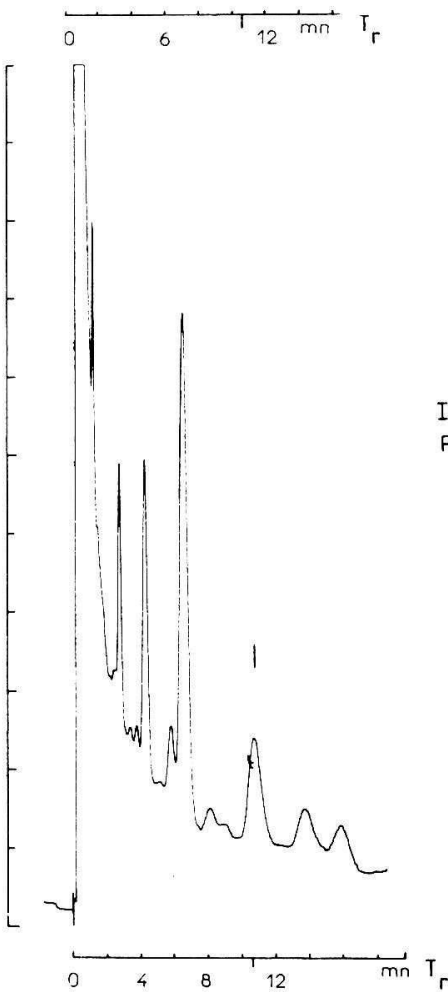

FIG. 1: Analyse en chromatographie en phase gazeuse des échantillons méthylés d'acide abscisique et d'inhibiteur extrait de Pinus sylvestris. A gauche, sur colonne $2 \% Q F-1$; à droite, sur colonne $2 \%$ $S E-33$ 
croissance aussi bien que ceux des inhibiteurs. De fait, Michniewicz (1967) trouve que, chez le pin, la baisse du taux des inhibiteurs s'accompagne d'une augmentation de celui des gibbérellines endogènes. La croissance et la dormance des conifères sont probablement sous la dépendance d'un équilibre hormonal dans lequel l'acide abscisique joue un rôle qui, pour n'être pas unique, n'en est pas moins important.

Reçu pour publication en janvier 1970.

\title{
REMERCIEMENTS
}

Je remercie M. J.-P. Nitsch ainsi que M. H. Harada qui, tant sur le plan théorique que pratique, m'ont guidé avec beaucoup de patience dans ce travail. Je tiens aussi à remercier tout spécialement M. B. H. Most qui a bien voulu réaliser pour moi l'indispensable et ultime analyse de chromatographie en phase gazeuse.

\section{SUMMARY \\ IDENTIFICATION OF ABSCISIC ACID IN DORMANT BUDS OF Pinus sylvestris}

Dormant buds of Pinus sylvestris L. contain a substance inhibitory to excised wheat coleoptiles. After purification by liquid-liquid partitioning at various $\mathrm{pH}$ 's, thin-layer chromatography, the purified extract has been found to contain, after methylation and gas chromatography on two different types of columns a peak at the position of abscisic acid. According to calculations based on the biological activity of the extracts, the content of inhibitor in dormant buds should be in the neighbourhood of $4 \mathrm{mg}$ of $\mathrm{ABA}$ per $\mathrm{kg}$ fresh weight.

\section{ZUSAMMENFASSUNG}

\author{
IDENTIFIZIERUNG DER «ABSCISIC ACID» BEI DEN SCHLAFENDEN KNOSPEN \\ vON Pinus sylvestris.
}

Während des Ausbrechens der Knospen nimmt die Konzentration des Wachstumshemmenden Faktors bei den schlafenden Knospen von Pinus sylvestris wesentlich ab. Die physiologischen und chromatographischen Eigenschaften dieses Hemmungsfaktors sind denen der « abscisic acid» ähnlich.

Nach wiederholter Saülen-und Dünnschichtchromatographie, hat sich der Hemmungsfaktor bei Gasphase-Chromatographie als dem «abscisic acid» ähnlich erwiesen. Eine auf biologische Versuchen gegründete Schätzung zeigt, dass die Konzentration von «abcisic acid» während der Winterruhe relativ hoch ist (etwa $4 \mathrm{mg} / \mathrm{kg}$ frischer Substanz).

\section{REFERENCES BIBLIOGRAPHIQUES}

Addicotr F. T., 1963. On the physiology of abscisin. In: «Régulateurs naturels de la croissance végétale ». Coll. Int. C.N.R.S., n० 123. Gif sur-Yvette, 687-703.

Bonnet-Masimbert M., 1968. Acide abscisique et dormance chez les végétaux ligneux. D.E.A. Fac. Sci., Paris.

CORnforth J. W., 1965. Chemistry and physio'ogy of «Dormins » in sycamore. Identity of sycamore dormin with Abscisin II. Nature, G.B., 205, 1269. 
Davis L. A., 1968. Gas-liquid chromatography of trimethylsilyl derivatives of abscisic acid and other plant hormones. Plant Physiol., 43, 1389-1394.

Mac Millan J., Pryce R. J., 1968. Recent studies of endogenous plant growth substances using combined gas chromatography, mass spectometry. Monograph 31. S.I.C. (14 Belgrave Square, London sw), 36-50.

MichNiewiCz M., 1967. The dynamics of gibberellin-like substances and growth inhibitors in ontogeny of conifers. Wissench. Z. Univ. Rostock Math.-Naturw. Reihe, 16, 577-583.

Milborrow B. V., 1967. The identification of $(+)$ Abscisin II [ + - - Dormin] in plants and measurement of its concentrations. Planta (Berl.), 76, 93-113.

Most B. H., 1969. The growth inhibitors of sugar cane. XI' Congr. intern. Bot., Abstracts, p. 153.

Nitsch J. P., NitsCh C., 1956. Studies on the growth of coleoptile and first internode sections. A new sensitive straight growth test for auxins. Plant Physiol., 31, 94-111.

Nitsch J. P., NitsCH C., 1959. Photoperiodic effects in woody plants: evidence for the interplay of growth-regulating substances. In: Photoperiodism and related phenomena in plants and animals, R.B. Withrowed, A.A.A.S., Washington, D.C., p. 225-242.

Rudnicki R., 1969. Studies on abscisic acid in apple seeds. Planta (Berl.), 86, 63-68.

Wareing P. F., Eagles G. F., Robinson P. M., 1963. Natural inhibitors as dormancy agents. In : «Régulateurs naturels de la croissance végétale ». Colloque intern. C.N.R.S. (123), Gif-surYvette, 377-386.

Wareing P. F., El. Antalbly H. M. M., Good J., Manuel J., 1967. The possible role and mode of action of abcisin (dormin) in the regulation of plant growth and development. Wissensch. Z. Univ. Rostock Math.-Naturw. Reihe, 16, 667-672. 